\title{
Energía a partir de residuos sólidos urbanos, caso parroquia Limoncocha en la Amazonía ecuatoriana
}

Energy from Solid Urban Waste, Case of the Limoncocha Parish in the Ecuadorian Amazon

Energia a partir de resíduos sólidos urbanos, o caso da parroquia Limoncocha na Amazônia equatoriana

\author{
Katty Coral Carrillo \\ Universidad Internacional SEK. Quito, Ecuador \\ katty.coral@uisek.edu.ec \\ https://orcid.org/0000-0002-1680-5650 \\ Jorge Oviedo Costales \\ Universidad Internacional SEK. Quito, Ecuador \\ jorjandrito@hotmail.com \\ https://orcid.org/0000-0002-8397-3419 \\ Ana Rodríguez Machado \\ Universidad Politécnica del Carchi. Tulcán, Ecuador \\ ana.rodriguez@upec.edu.ec \\ https://orcid.org/0000-0002-7348-6094
}

DOI: https://doi.org/10.32719/25506641.2021.9.9

Recibido: 21 de mayo de 2020 • Revisado: 14 de julio de 2020 • Aceptado: 27 de agosto de 2020 Artículo de investigación

Licencia Creative Commons

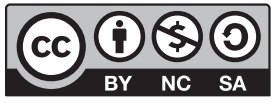




\section{Katty Coral Carrillo, Jorge Oviedo Costales y Ana Rodríguez Machado}

\section{Resumen}

La sobreproducción de residuos sólidos urbanos (RSU) aqueja tanto al ambiente como a la salud humana; la parroquia Limoncocha, ubicada en la Amazonía ecuatoriana, no es la excepción, disponiendo sus residuos en un botadero de basura ubicado en el cantón Shushufindi o tomando sus propias medidas, disponiendo los residuos en fosas domiciliarias o mediante quema al aire libre. El estudio forma parte de una investigación sobre las propiedades químicas, físicas y biológicas de los RSU, una de ellas, su uso como fuente potencial de energía, utilizando procesos de aprovechamiento energético. Se caracterizan las concentraciones de residuos de comida, plásticos, papel, cartón y en menor cantidad de madera y textiles. Se evaluó la posibilidad de aprovechamiento energético mediante la incineración a través de poder calórico superior (PCS) e inferior (PCI) que podrían ser recuperados, obteniendo valores entre 3154 y $4560 \mathrm{kcal} / \mathrm{kg}$ para PCS y 3100 y 4436 $\mathrm{kcal} / \mathrm{kg}$ para PCI. Los resultados indican que el $92 \%$ en peso contienen potencial energético superior a $3100 \mathrm{kcal} / \mathrm{kg}$, pudiendo ser sometidos a procesos de aprovechamiento energético. Para su uso es necesaria la formación de una mancomunidad con las poblaciones aledañas, ya que el flujo de residuos que equivale a 1,7 t / día es insuficiente para implementar dicho sistema individualmente.

Palabras clave: residuos sólidos, contaminación, poder calórico, incineración, valorización energética.

JEL: Q01 Desarrollo sostenible.

\section{Abstract}

The solid urban waste overproduction (MSW) affects both the environment and human health; the Limoncocha parish, located in the Ecuadorian Amazon Region is no exception, disposing its waste in a garbage dump located in Shushufindi or taking its own measures, disposing the waste in household pits or by burning in the open air. The study is part of an investigation on the chemical, physical and biological properties of MSW, properties like, its use as a potential source of energy using energy harvesting processes. The concentrations of food waste, plastics, paper, cardboards and in a smaller quantity, wood and textiles are characterized. The possibility of energy use was evaluated through incineration among higher (PCS) and lower (PCI) caloric power that could be recovered, obtaining values between 3154 and $4560 \mathrm{kcal} / \mathrm{kg}$ for PCS and 3100 and $4436 \mathrm{kcal} / \mathrm{kg}$ for PCI. The results indicate that $92 \%$ by weight contains potential energy greater than $3100 \mathrm{kcal} / \mathrm{kg}$ and can be subjected to energy use processes. In order to be used, it is necessary to create a community with the surrounding populations since the waste flow that is equivalent to $1.7 \mathrm{t} /$ day is insufficient to implement said system individually.

Keywords: Solid waste, pollution, caloric power, incineration, energy recovery.

JEL: Q01 Sustainable development. 


\section{Resumo}

A sobreposição de resíduos sólidos urbanos (RSU) afeta tanto o ambiente quanto a saúde humana. A parroquia (divisão político-territorial utilizada no Equador) Limoncocha, localizada na Amazônia equatoriana, não é exceção, dispondo seus resíduos em um depósito de lixo localizado no cantón (divisão administrativa de segundo nível, logo após os estados) Shushufindi ou tomando medidas próprias, descartando os resíduos em fossas domiciliares ou queimando-os ao ar livre. Este estudo forma parte de uma pesquisa sobre as propriedades químicas, físicas e biológicas dos RSU, sendo uma delas seu uso como fonte potencial de energia por meio de processos de aproveitamento energético. Foram caracterizadas as concentrações de resíduos de comida, plásticos, papel, papelão e, em menor quantidade, madeira e resíduos têxteis. Avaliou-se a possibilidade de aproveitamento energético por meio da incineração através de poder calorífico superior (PCS) e inferior (PCI), os quais poderiam ser recuperados, obtendo-se valores entre 3154 e 4560 $\mathrm{kcal} / \mathrm{kg}$ para PCS e entre 3100 e $4436 \mathrm{kcal} / \mathrm{kg}$ para PCI. Os resultados indicam que $92 \%$ em peso contêm potencial energético superior a $3100 \mathrm{kcal} / \mathrm{kg}$, podendo ser submetidos a processos de aproveitamento energético. Para seu uso, é necessária a formação de uma entidade intermunicipal com as populações aldeãs, uma vez que o fluxo de resíduos equivalente a 1,7 t/ dia é insuficiente para implementar tal sistema individualmente.

Palavras-chave: Resíduos sólidos, contaminação, poder calorífico, incineração, valorização energética.

JEL: Q01 Desenvolvimento sustentável.

\section{Introducción}

L

os residuos sólidos urbanos (RSU) son producto, tanto de actividades humanas domésticas como de actividades comerciales e industriales, por lo cual se busca reducir su generación en la fuente, y solo cuando los materiales no puedan ser reciclados se considera alguna estrategia de recuperación de los residuos entre los que resaltan mecanismos térmicos de aprovechamiento energético como la incineración (Moratorio, Rocco y Castelli, 2012; Banco Mundial 2018).

Ecuador, al 2010, presentaba una población de aproximadamente $16 \mathrm{mi}$ llones de habitantes que generaban 11.200 toneladas de RSU por día (INEN 2014), con apenas un $39 \%$ de disposición en rellenos sanitarios, en tanto que la restante cantidad de residuos se deposita en botaderos a cielo abierto (23\%), botadero controlado (26\%) y un $12 \%$ en celda emergente (INEN 2014); por lo que resulta preciso realizar estudios que permitan determinar la factibilidad de la implementación de tecnologías de aprovechamiento de los RSU. 
En 2006, el "Programa de Diálogos de la iniciativa energética de la Unión Europea para el desarrollo sostenible" (EUEI-PDF) y la Secretaría Ejecutiva de la Organización del Tratado de Cooperación Amazónica (OTCA) decidieron promover el diagnóstico de la demanda rural de energía en la Amazonía. El propósito era identificar lineamientos de integración regional y de cooperación para promover al acceso efectivo de las sociedades rurales amazónicas a energía y desarrollo sostenible (EUEI 2008). En abril de 2010 se creó el Programa Nacional para la Gestión Integral de Desechos Sólidos (MAE-PNGIDS), cuya meta principal es impulsar la gestión de los residuos sólidos urbanos en los municipios del Ecuador, con un enfoque integral y sostenible, con la finalidad de disminuir la contaminación ambiental y mejorar la calidad de vida de la población del país, utilizando estrategias, planes y actividades de capacitación, sensibilización y estímulo a los diferentes actores relacionados (EC MAE 2015).

La decisión de incinerar residuos, en lugar de enviarlos a un vertedero, requiere de una cuidadosa consideración de criterios, así como de estudios técnicos, que implican desde la caracterización hasta estudios complejos de composición química y energética. La realidad latinoamericana al momento es que se están realizando varios esfuerzos para minimizar la generación de residuos sólidos urbanos; sin embargo, algunas de las actividades relacionadas con la reducción, recuperación y reciclaje se muestran incipientes.

La Reserva Biológica Limoncocha (RBL) se encuentra ubicada en el nororiente de la Amazonía ecuatoriana, en la región suroccidental de la provincia de Sucumbíos, cantón Shushufindi, parroquia Limoncocha, a una altura aproximada de $230 \mathrm{msnm}$, con una temperatura anual de $24,9^{\circ} \mathrm{C}$ y una precipitación anual que alcanza hasta los $3065 \mathrm{~mm}$ (Bastidas 2009 y Montenegro 2015).

Es un área protegida que coexiste con la comunidad del mismo nombre, misma que mantiene un permanente crecimiento demográfico y, consecuentemente, un incremento constante de la cantidad de RSU y como resultado, impactos sociales y ambientales. Debido a que la RBL se encuentra en una zona de gran biodiversidad y es sensible a alteraciones ambientales, es necesario implementar medidas para un adecuado manejo y aprovechamiento de los RSU (Cabrera 2016). 
De acuerdo con el censo de 2010 (INEC 2010), la parroquia Limoncocha contaba con 6817 habitantes con una tasa de crecimiento del 3,48\%, y está conformada por 7146 habitantes, dispuestos en tres asentamientos principales; Limoncocha, Yamanunka y 18 de Noviembre. En cuanto a la distribución étnica de la población, el 43,39\% se califica como mestizo mientras que el $51,56 \%$, se considera indígena cuyos principales grupos son el quichua y el shuar.

Dentro de la parroquia Limoncocha, el Gobierno Autónomo Descentralizado del cantón Shushufindi es el ente competente para la recolección y posterior tratamiento de RSU que genera dicha localidad. El sitio de disposición final de los residuos es un relleno sanitario precariamente manejado, ubicado en el cantón Shushufindi.

Apenas el 13,30\% de los habitantes de la parroquia tienen acceso al servicio de recolección de basura, el 29,06 \% eliminan la basura en terrenos baldíos, mientras que el 30,66\% los quema, el resto de habitantes opta por otras formas de eliminación, como enterrar o arrojar a ríos (Marañón 2015).

Actualmente no existe ningún programa concreto por parte del Estado para el aprovechamiento energético de los residuos sólidos urbanos en la región amazónica ecuatoriana ni en la localidad de Limoncocha. A partir del año 2015 se dio inicio al estudio de determinación del PCS y PCI de los RSU en la parroquia Limoncocha, lo que permitió tener pautas para dar seguimiento a dicha investigación. Las muestras fueron tomadas mensualmente hasta 2017, obteniendo un total de 32 muestras en las que se realizaron estudios de composición, relación $\mathrm{C} / \mathrm{N}$, porcentaje de cenizas, metales pesados en cenizas, entre otros, que complementan al presente, con la finalidad de plantear opciones al gobierno parroquial para un adecuado manejo y gestión de los RSU.

La parroquia Limoncocha, como se aprecia en gráfico 1, representa un sitio estratégico desde el punto de vista ambiental, ya que aloja un sinnúmero de especies, tanto animales como vegetales; a más de ello, aquí se encuentra la laguna Limoncocha, catalogada como un sitio de humedales de importancia internacional, según la convención de RAMSAR, ocupando el segundo lugar en importancia de humedales del país (Montenegro 2015).

La parroquia también alberga una población en constante crecimiento (ASOKIL 2015); de acuerdo con el INEN (2014), el Ecuador tiene un crecimiento poblacional del $1,4 \%$ anual, lo cual repercute en un incremento, al 
menos proporcional, de la generación de RSU. Como consecuencia, estudiar tanto el flujo como las características fisicoquímicas de los RSU, a fin de establecer las condiciones óptimas para implementar un sistema de gestión que posibilite la conservación de las características naturales del sitio, a más de sostener una localidad limpia y una población sana.

La incineración es uno de los procesos térmicos que pueden aplicarse en el tratamiento de los RSU para disminuir su cantidad y aprovechar su energía (Muñoz 2019). Para el manejo de los RSU, una de las mejores alternativas es el aprovechamiento energético, constituyéndose en una herramienta que permite evitar que los RSU contaminen en gran escala al ambiente, a la par de obtener un beneficio económico (López, Álvarez y Alguacil 2012).

Debido a las ventajas que presenta el mecanismo de incineración de RSU, el cual manifiesta no solo una mejora en el sistema de gestión y reducción de espacios para rellenos sanitarios, sino también permite aprovechar la energía generada al combustionar los mismos, resulta importante realizar la investigación de cuantificación del poder calorífico superior (PCS) y poder calorífico inferior (PCI), ya que el buen resultado de un proyecto de aprovechamiento energético de residuos depende, en primer lugar, de datos exactos sobre las futuras cantidades de residuos y las características de los mismos. Con la información recopilada se podrán establecer políticas y diseñar un sistema de gestión adecuado para las zonas de características similares a la comunidad de Limoncocha.

El poder calorífico es el parámetro fundamental para el desarrollo de procesos de recuperación energética, siendo de vital importancia el porcentaje de cenizas producido. Adicionalmente, se deben conocer características como la eventual presencia de productos tóxicos, metales pesados, contenido de elementos inertes, entre otros, información que permite diseñar soluciones apropiadas en los procesos de recuperación y para establecer las adecuadas precauciones higiénicas y sanitarias (Moratorio 2012). El poder calorífico de un material combustible es una característica intrínseca que representa la cantidad de calor desprendida en la combustión completa por unidad de masa, y sus expresiones más comunes son: kcal/kg y BTU / lb (Barras 2008).

La conversión térmica de los RSU puede darse de tres formas: combustión, gasificación y pirólisis. En la combustión y en la gasificación la reacción química que se produce es exotérmica, lo que significa que se libera 
energía directamente. Sin embargo, en la gasificación se obtiene un producto gaseoso con un poder calorífico remanente que luego puede ser aprovechado en una combustión posterior (Moratorio, Rocco y Castelli 2012).

En la determinación del poder calorífico de los residuos se pueden utilizar los métodos analítico y práctico o experimental. El método analítico permite determinar el poder calórico del residuo basándose en fórmulas, mientras que el método experimental utiliza una bomba calorimétrica adiabática, que se utiliza para determinar el poder calórico de una muestra seca de residuo.

\section{Metodología}

El método para esta investigación es el inductivo-deductivo, ya que, al analizar muestras representativas, tomadas directamente de los distintos sitios de depósito de la parroquia, es posible inferir la cantidad de poder calórico general de los RSU para la zona urbana de Limoncocha.

\section{Tamaño de muestra}

Para establecer los días de muestreo se utilizó la fórmula de estadística aplicada a poblaciones finitas. Se consideró como espacio temporal los 12 meses del año. El nivel de confianza fue del $95 \%$, lo cual es representativo para la muestra de la población, dando como valor $\mathrm{z}=1,96 \%$, en torno al error relativo a aceptar, que es del $10 \%(\mathrm{e}=0,1)$

Se utilizó la fórmula planteada por Morales (2012):

$$
\mathrm{n}=\frac{\mathrm{N}}{1+\frac{\mathrm{e}^{2}(\mathrm{~N}-1)}{\mathrm{Z}^{2} \mathrm{pq}}}
$$

Donde:

$\mathrm{n}=$ tamaño de la muestra que se desea conocer.

$\mathrm{N}=$ tamaño conocido de la población.

$\mathrm{z}=$ valor de $\mathrm{z}$ correspondiente al nivel de confianza. 
Un nivel de confianza del $95 \%$ (también expresado como: $\alpha=, 05$ ) corresponde a $\mathrm{z}=1,96$ sigmas o errores típicos; $\mathrm{z}=2$ (dos sigmas) corresponde a un $95,5 \%$ (aproximadamente, $\alpha=, 045$ ).

$\mathrm{pq}=$ varianza de la población.

La varianza en los ítems dicotómicos (dos respuestas que se excluyen mutuamente) es igual a pq, y la varianza mayor (la mayor diversidad de respuestas) se da cuando $\mathrm{p}=\mathrm{q}=0,50$ (la mitad de los sujetos responde sí y la otra mitad responde no), por lo que en esta fórmula [1] pq es siempre igual a $(0,50)(0,50)=0,25$ (es una constante) .

$\mathrm{e}=$ error muestral.

Los muestreos en la RBL se realizaron una vez al mes, durante una semana por 11 meses; la semana escogida correspondió a una selección aleatoria completa. Al tratarse de dos años y medio de muestreo se recolectaron un total de 31 muestras (Pacheco 2016).

Mazzilli (2014) establece, para el DMQ, resultados de reducción de volumen en base húmeda, concordantes con la teoría que presenta a la incineración como una alternativa para minimizar los volúmenes de residuos a disponer. Los valores indican un porcentaje general de reducción del 92,34\%, dato corroborado por Garcés (2016), pudiendo llegarse a valores similares para la parroquia Limoncocha.

\section{Descripción del área de investigación}

En la cabecera parroquial de Limoncocha habitan 874 personas. La principal actividad económica de la zona es la agricultura, dedicándose a esta actividad el $33 \%$ de la población. Los principales productos cultivados son: plátano, yuca, café, cacao y maíz. Si bien la mayor parte de productos son para el consumo familiar, ocasionalmente se produce el intercambio con otros comuneros o se los comercializa en las ferias locales (Marañón 2015).

Del reconocimiento realizado a la zona se observó una estructura urbanizada de la parroquia Limoncocha que tiende a la conformación típica de una pequeña ciudad, con lotización de terrenos dentro de un diseño de manzanas, con calles, canchas para la recreación de la población, comercios, casa comunal, infocentro, escuela, colegio y hoteles comunitarios. A pesar de su reducido tamaño, la parroquia cuenta con servicios como luz y agua potable, 
cuya disponibilidad constituye un atractivo para la población, razón por la cual varias familias del área rural y circundante se trasladaron a vivir en esta área urbana, mejorando las condiciones para el comercio y el intercambio de bienes, pero incrementando las necesidades de gestión adecuada de residuos. Los RSU de Limoncocha, al igual que cualquier recinto urbano, pueden ser aprovechados para generación de energía a través de procesos bioquímicos tales como la biodegradación aerobia o anaerobia, y/o procesos de oxidación termoquímica como la incineración, gasificación y/o pirolisis. Estos últimos poseen un mayor potencial energético y mayor capacidad de reducción del volumen de RSU (Montiel-Bohórquez y Pérez 2019).

Los residuos tienen una cantidad mayoritaria de materia orgánica (restos de alimentos, material de poda, eses de animales, etcétera), siendo esta del 73 \% (Mora 2016). El porcentaje de textiles es superior al de papel y cartón; a pesar de ello, los textiles no se generan de forma constante. En cuanto a la producción de plásticos, esta incrementa considerablemente durante las fechas festivas de la parroquia debido a su uso en la comercialización de alimentos. La producción de basura en Limoncocha es de 0,61 kg / hab. / día. De encuestas realizadas en el proyecto de Mora (2016), se determinó que el volumen de residuos que se someten a quema a cielo abierto es de alrededor del $30 \%$.

El sistema de recolección denominado "a pie de vereda", que se realiza en la parroquia, dificulta el aprovechamiento, ya que al no estar separados los RSU, se modifican las condiciones propias del residuo. Adicionalmente, la recolección de residuos dos veces por semana provoca que la población busque formas de deshacerse de sus residuos, especialmente los sanitarios, por lo que recurre a la quema a cielo abierto, provocando gases de combustión, con el peligro consecuente de la generación de dioxinas y furanos.

\section{Muestreo}

Para llevar a cabo el proceso de determinación del PCI y PCS se tomaron muestras mensuales de los RSU en la parroquia Limoncocha a partir de agosto de 2015, de forma mensual hasta agosto de 2017. Estas muestras fueron trasladadas al laboratorio de procesos de la Universidad Internacional SEK (UISEK) para su análisis. Durante esta etapa se procesaron 31 muestras 
de papel, cartón, madera, textiles materia orgánica y mixto (combinación proporcional de todos los residuos en las proporciones correspondientes a la caracterización), obteniéndose la cantidad de energía en $\mathrm{kcal} / \mathrm{kg}$ de estos residuos al someterlos a un proceso de combustión en una bomba calorimétrica de Parr.

Los resultados obtenidos son producto de un monitoreo constante a lo largo de 31 meses en los años 2015, 2016 y 2017. La línea de investigación de la UISEK ha planteado varios estudios complementarios al presente estudio, tanto en la comunidad de Limoncocha como en el distrito.

En cuanto a la fase de campo, la metodología establecida como base para el muestreo de RSU fue el método sencillo del análisis de RSU del Centro Panamericano de Ingeniería Sanitaria y Ciencias del Ambiente (CEPIS), desarrollada por Sakurai en 2000. La metodología indica que pesadas, hasta conseguir $50 \mathrm{~kg}$ de RSU. Una vez recolectado el peso deseado se traslada a un sitio de preferencia pavimentado, en donde se vierte y se forma un montículo de residuos. Posteriormente se realizan tres cuarteos, obteniéndose finalmente una muestra representativa de aproximadamente seis kilogramos de RSU (CEPIS 2000), como se muestra en el gráfico 1.

\section{Gráfico 1}

\section{Método de muestreo por cuarteo}

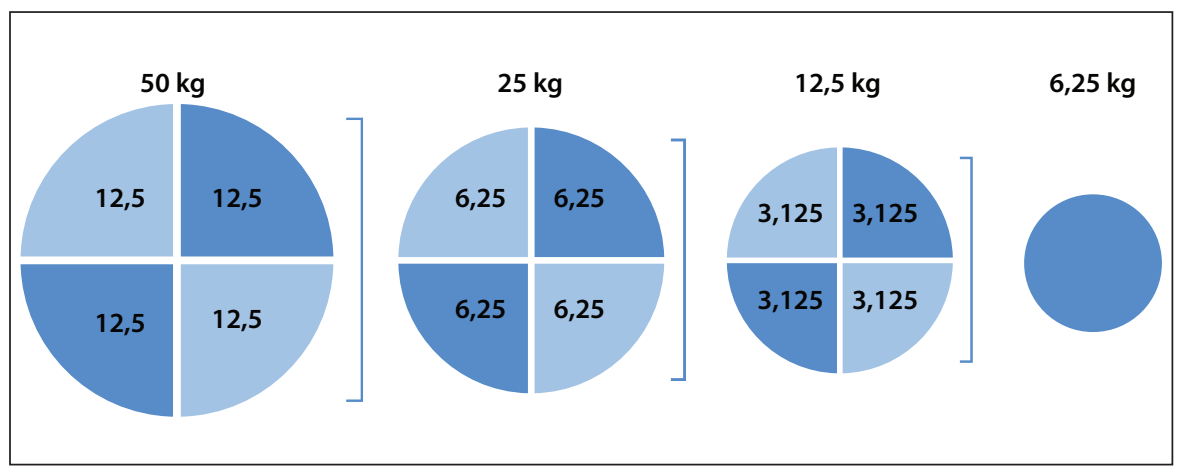

Fuente: Ninabanda (2016). 


\section{Caracterización de los RSU de la parroquia Limoncocha}

En la última porción del cuarteo se clasifican los residuos en textiles, papel, cartón, madera, materia orgánica y otros (aquellos que no corresponden a ninguna de las categorías previamente clasificadas). Esta clasificación es manual, luego de lo cual se pesan las diferentes categorías y se determina el porcentaje en peso de cada una. Este proceso se lo realiza paralelamente a un muestreo in situ en una muestra representativa de la población; de esta manera se obtienen datos validados por las dos metodologías.

\section{Análisis de PCS y PCI}

En la fase de laboratorio se realizó una homogeneización y picado de las muestras en trozos de tamaño inferior a un centímetro; las muestras de textiles, papel, cartón, madera y materia orgánica fueron tratadas como puras, en tanto que la muestra mixta fue preparada con cada uno de los residuos proporcionalmente a la caracterización de los residuos realizada previamente

Las muestras fueron sometidas a un proceso de secado en una estufa durante 24 horas, a una temperatura de $105^{\circ} \mathrm{C}$, con el fin de eliminar la humedad contenida en los residuos. Para el cálculo del porcentaje de humedad se aplicó la fórmula establecida por el laboratorio de Suelos y Agua de Sáenz Peña (2005).

$$
\begin{gathered}
\% \mathrm{H}=\text { peso de la muestra húmeda }- \text { peso de muestra seca } \\
\text { peso de muestra húmeda } * 100
\end{gathered}
$$

Una vez seca la muestra, se procedió a pelletizarla; ${ }^{1}$ dicho pellet fue procesado en una bomba calorimétrica de Parr, equipo que brinda la posibilidad de determinar el PCS a través de la fórmula establecida por el manual de instrucciones para su funcionamiento (código del manual: No. 204M.).

$$
\mathrm{PCS}=(\mathrm{tW}-\mathrm{e} 1-\mathrm{e} 2-\mathrm{e} 3) \mathrm{m}
$$

1. Pelletizar: formar un pellet, en este caso compactar una muestra de residuo para formar un cilindro de un centímetro de radio y altura. 
En donde:

PCS = calor de combustión (cal / g).

$\mathrm{t}=$ aumento de temperatura $\left({ }^{\circ} \mathrm{C}\right)$.

$\mathrm{W}=$ constante del equipo $\left(\mathrm{cal} /{ }^{\circ} \mathrm{C}\right)$.

$\mathrm{m}=$ masa de la muestra $(\mathrm{g})$.

$\mathrm{e}_{1}=$ corrección en calorías por el calor de formación de ácido nítrico.

$\mathrm{e}_{2}=$ corrección en calorías para el calor de formación de ácido sulfúrico. ción.

$\mathrm{e}_{3}=$ corrección en calorías por el calor de combustión del cable de igni-

El cálculo del PCI se realizó por medio de una fórmula establecida por Sakurai (2000).

$$
\mathrm{PCI}=\mathrm{PCS}-\% \mathrm{H} 100 * 600
$$

En donde:

$\mathrm{PCS}=$ poder calórico superior (cal / g).

$\mathrm{PCI}=$ poder calórico inferior (cal / g).

$\% \mathrm{H}=\%$ humedad.

\section{Resultados}

\section{Caracterización de los residuos de la parroquia Limoncocha}

Para este estudio de aprovechamiento energético, se desecharon aquellos materiales que no disponen de poder calórico, tales como el vidrio, la tierra o los metales. 
Gráfico 2

Caracterización de los residuos de la parroquia Limoncocha

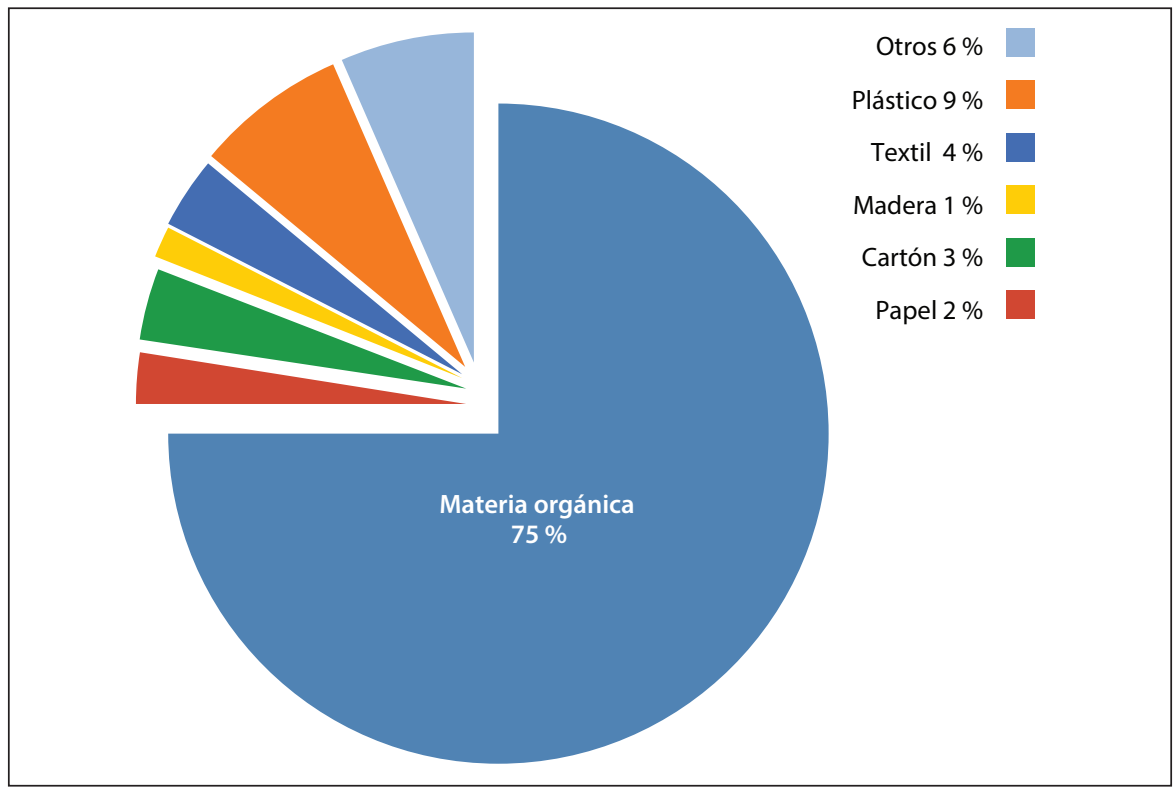

Elaboración propia.

Gráfico 3

Desviación estándar de la caracterización de RSU

de la parroquia Limoncocha

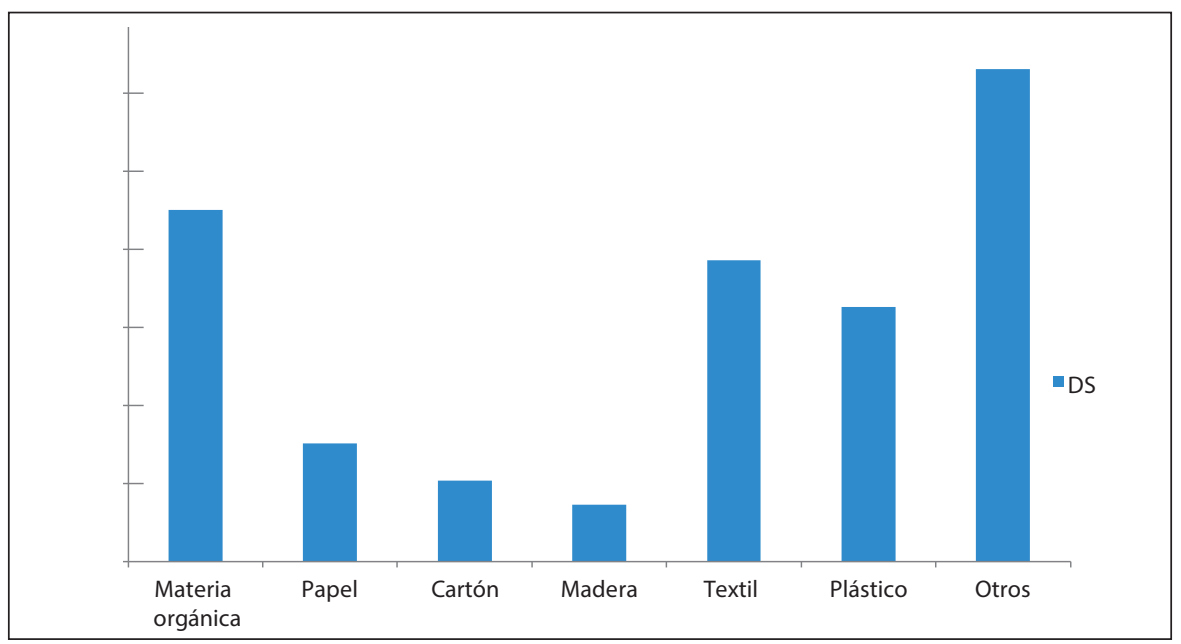

Elaboración propia. 
El gráfico 2 muestra la caracterización de los RSU de la parroquia Limoncocha, en tanto que en el gráfico 3 se presenta la desviación estándar de los datos de dicha caracterización, obtenida de las 31 muestras, evidenciándose el mayor valor, en la muestra correspondiente a "Otros", esto debido a la variedad de residuos que involucra esta categoría y a las diferentes disposiciones de esta tipología de RSU. A esta categoría pertenecen residuos como los sanitarios, pañales desechables en la mayoría de días de muestreo, aunque en cantidades inferiores al $1 \%$, mientras que el resto de este tipo de residuos se pudo verificar in situ, que son quemados a cielo abierto en los mismos domicilios o arrojados en fosas, quebradas o terrenos baldíos, lo que repercute en la calidad ambiental de la zona, sobre todo en el medio paisajístico, pues al tratarse de una reserva biológica, todo residuos fuera de lugar genera malestar en los turistas que visitan la zona.

\section{Humedad de los residuos de la parroquia Limoncocha}

De acuerdo con Alonso, Martínez y Olías (2003), y Lomas et al. (2001), el porcentaje de humedad óptimo que deben tener los RSU para ser aprovechados energéticamente es del 55 al $60 \%$. En el gráfico 4 se puede apreciar que los residuos de materia orgánica y mixto (mezcla de todos los residuos) no podrían ser sometidos a la valorización energética, sin previamente efectuar un proceso de secado, en tanto que papel, cartón, madera y textiles presentan valores de humedad acordes con el aprovechamiento energético. La humedad varía en función de la lluvia a la que se encuentra expuesta la basura previa a su recolección, porque se la depone, para su recolección en lugares abiertos; el nivel de lluvia en la zona en donde se recogió las muestras es de aproximadamente $3100 \mathrm{~mm} / \mathrm{m}$ (Climate-data 2018). Los porcentajes de humedad $(\% \mathrm{H})$ obtenidos en los RSU son un promedio de las 31 muestras y se presentan en el gráfico 5 , tanto en promedio general como anual.

\section{Poder calórico superior e inferior}

Los resultados obtenidos en cuanto a PCS y PCI de los RSU de la parroquia Limoncocha indican que los textiles y la madera son aquellos con 
Porcentaje de humedad de los residuos de la parroquia Limoncocha, comparativo anual

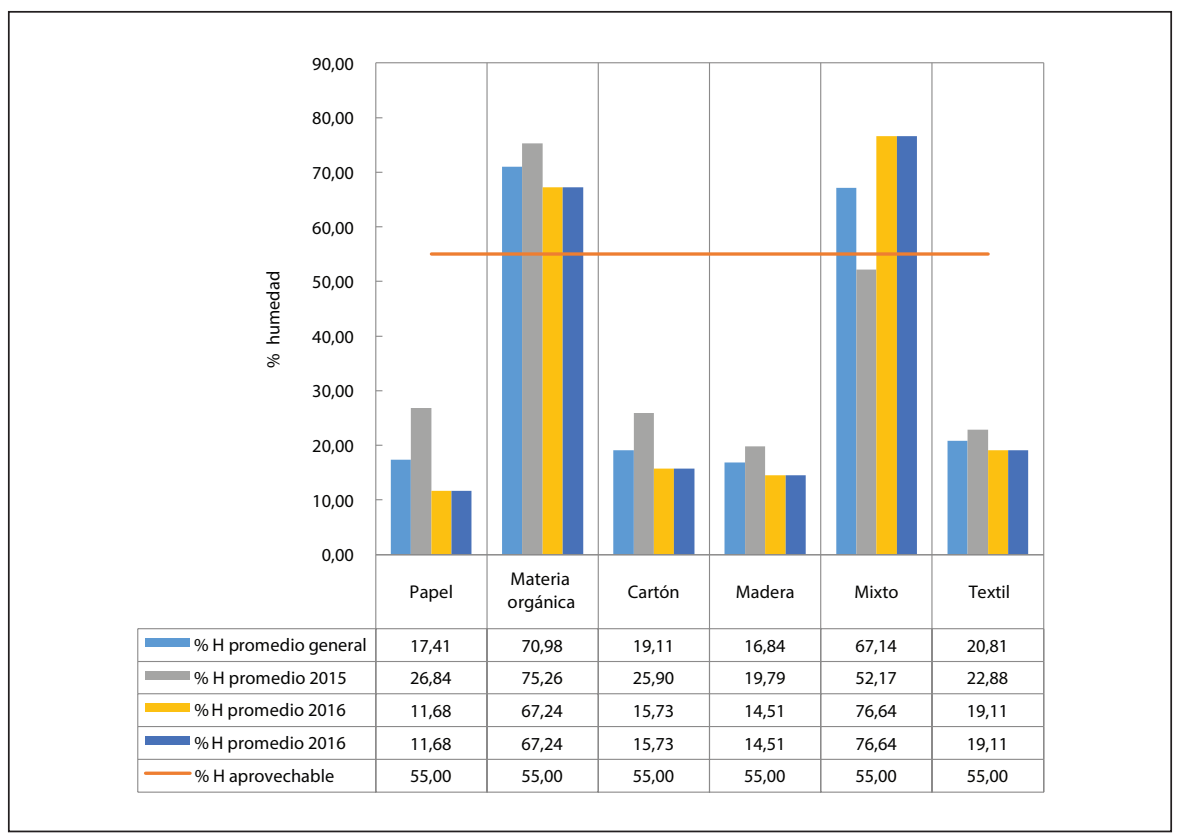

Elaboración propia.

mayor capacidad de generar calor, en tanto que el residuo con menor PCS y PCI es el papel.

La muestra mixta fue analizada de acuerdo a la composición de los residuos sin previa clasificación, es decir, tal como se la encuentra en el depósito de residuos de la parroquia.

Al ser el PCI uno de los parámetros más significativos a considerar para la implementación de un sistema de incineración de residuos, Romero (2010) indica que para que una planta incineradora alcance un rendimiento energético de entre el 20 y $30 \%$, el valor de PCI de los RSU debe ser de aproximadamente $3100 \mathrm{kcal} / \mathrm{kg}$. Como se puede apreciar en el gráfico 5, los RSU de la parroquia Limoncocha pueden ser considerados para aprovechamiento energético, desde el punto de vista de poder calórico exclusivamente, pues desde la materia orgánica hasta los textiles superan el PCI recomendado por el autor. 
Poder calórico superior e inferior de los RSU de la parroquia Limoncocha

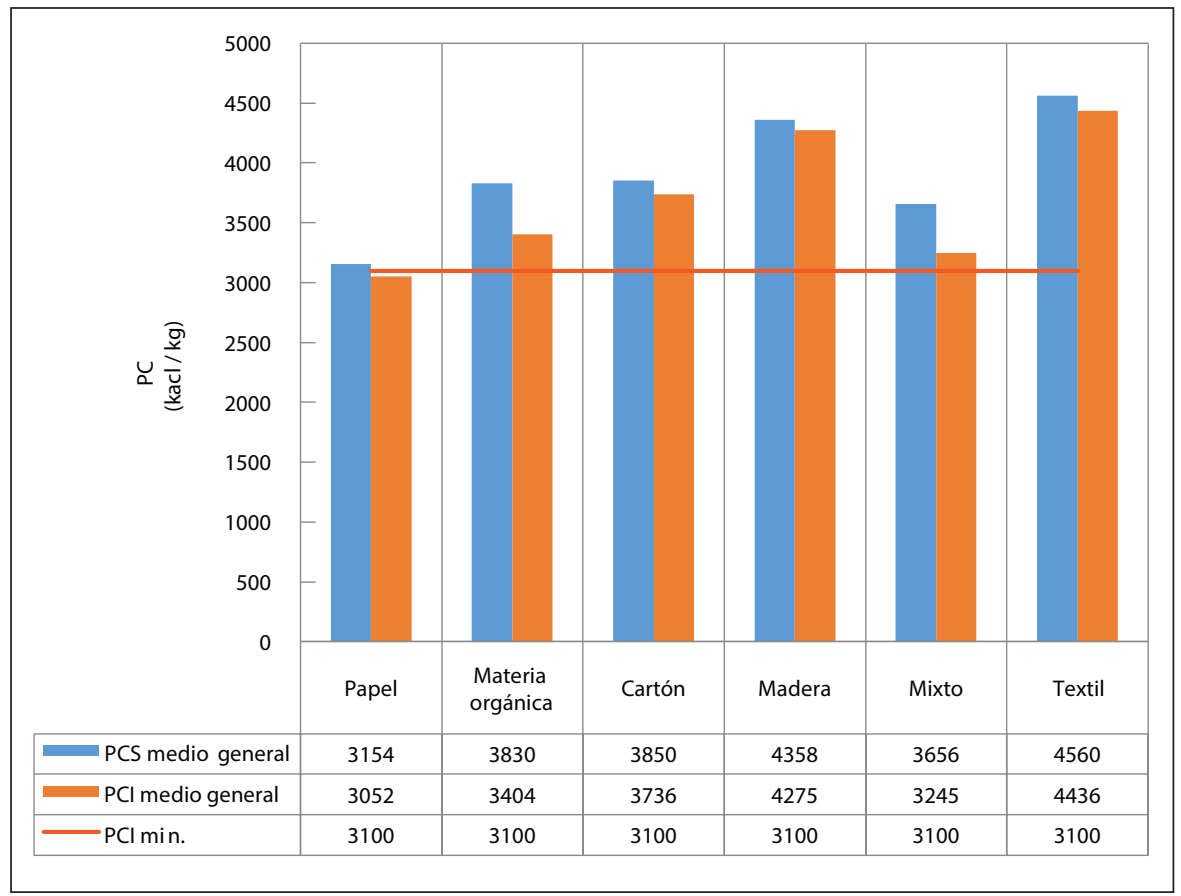

Elaboración propia.

En el gráfico 6 se aprecia que la mayor desviación está presente en el PCS papel, y la menor desviación en el PCI del mismo residuo, lo que se explica debido a la humedad que acumula el papel, al ser depositado junto a otro tipo de residuos, es tan alta que estandariza el PCI del papel sin tomar en cuenta su estructura; al retirar la humedad, el PCS hace notoria los diferentes tipos de papel que maneja la comunidad.

\section{Discusión}

Los datos obtenidos presentan similitudes y diferencias con otros estudios de poder calórico realizados por la UISEK en el DMQ. La principal diferencia radica en la producción per cápita de residuos: DMQ 0,78 kg/ hab. / día (Castillo 2012) y Limoncocha 0,53 kg / hab. / día. Adicionalmente, 
Gráfico 6

Desviación estándar de los valores de poder calórico superior e inferior de los residuos sólidos urbanos de la parroquia Limoncocha

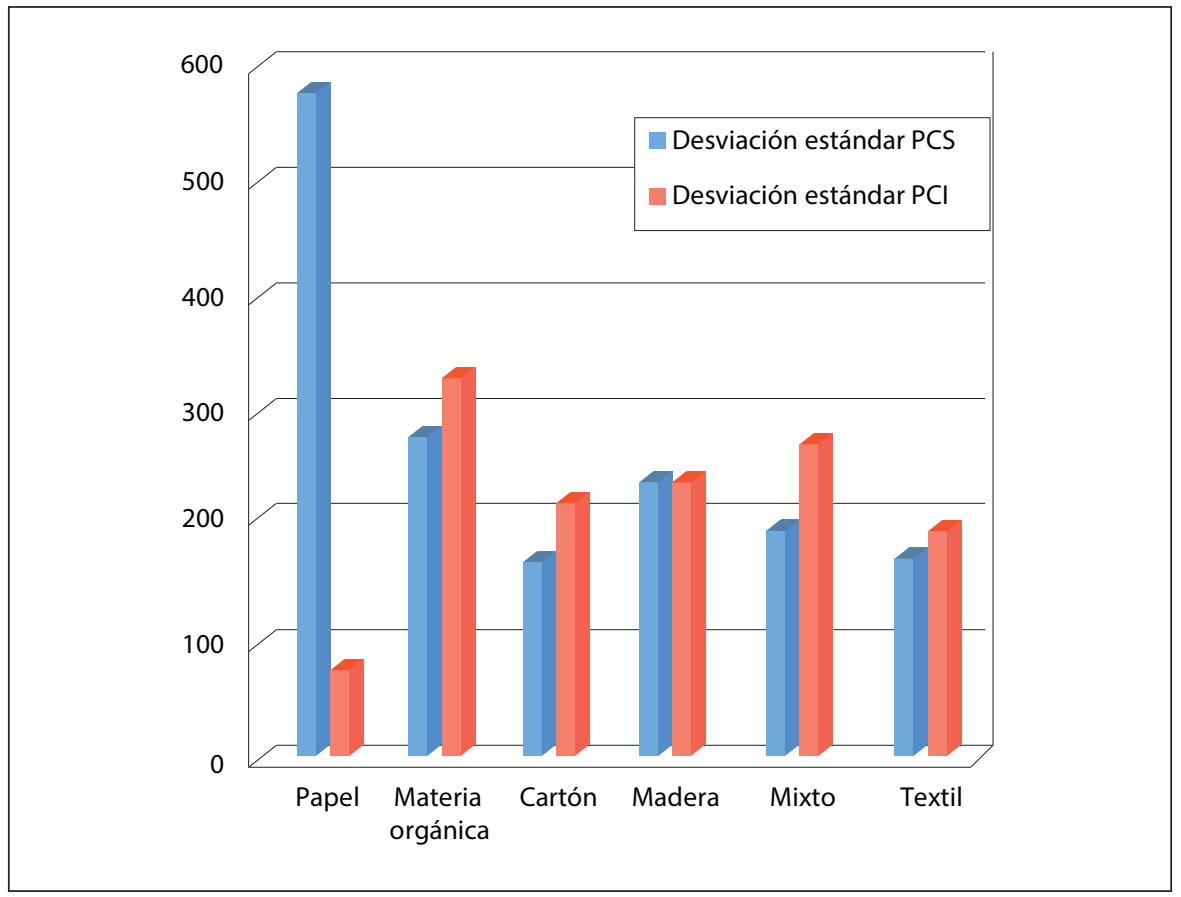

Elaboración propia.

la cantidad de materia orgánica (73\%), es mayor a la encontrada en el DMQ (63\%) (Ribadeneira 2014). En cuanto a la humedad, contrario a lo esperado, los residuos mixtos tanto del DMQ como de la parroquia Limoncocha tienen valores de humedad comprendidos entre un 60 y $68 \% \mathrm{H}$ (Mazzilli 2014).

El poder calórico superior de los RSU de Limoncocha $3656 \mathrm{kcal} / \mathrm{kg}$ es inferior a los del DMQ; Ayala (2013) establece, para el DMQ, valores que bordean las $4000 \mathrm{kcal} / \mathrm{kg}$. Esto se explica por la menor cantidad de residuos orgánicos de la ciudad versus los de la parroquia.

Los datos obtenidos proporcionan información indicativa de que un sistema de gestión integral de residuos no puede limitarse a un único sistema de tratamiento, como es el aprovechamiento energético planteado en este estudio, debiendo establecerse estudios complementarios para la prevención, 
minimización, reuso, reciclaje y posterior uso de tratamientos físicos, químicos, biológicos y térmicos de los RSU.

\section{Conclusiones}

La gestión de los RSU dentro de la parroquia Limoncocha es deficiente. No toda la cantidad de residuos es enviada al servicio de recolección parroquial, lo cual dificulta realizar un aprovechamiento energético; no se conocen los valores reales de generación de la parroquia, $(\sim 1,7 \mathrm{t} /$ día $)$, y se evidencia una inapropiada gestión de los residuos con su consiguiente repercusión en la calidad ambiental de la zona, por lo que se hace indispensable ubicar la mejor combinación de tratamientos para establecer el sistema de gestión de residuos óptimo.

Para un adecuado aprovechamiento de los RSU, la jerarquía de la gestión de residuos sólidos debe cumplirse de una forma que sea lo suficientemente flexible como para permitir al gobierno parroquial la implementación de instalaciones acordes con las condiciones locales y la capacidad operativa de los usuarios. Especial importancia tiene contar con el compromiso y la participación de toda la comunidad para que el sistema de gestión a implantarse, con base en los diferentes estudios realizados, pueda concretarse de manera eficiente. La sostenibilidad del proyecto se basará en una separación en la fuente y una recolección selectiva más frecuente, que permita un manejo integral de los mismos, preservando de esta manera el ecosistema frágil de la parroquia Limoncocha.

Debido a que la generación de residuos depende en gran medida de las condiciones socioeconómicas y el grado de urbanización y la industrialización del área intervenida (Banco Mundial 1999), se corrobora que los residuos de la parroquia rural Limoncocha están constituidos principalmente por residuos de comida provenientes de las viviendas y comedores. Los días festivos y el consumo de alimentos preparados incrementa notablemente la proporción de plásticos en los RSU de la comunidad.

Los hábitos de disposición de los residuos de los habitantes de la parroquia Limoncocha son un limitante para el aprovechamiento energético. El resultado de poder calórico superior e inferior de los RSU depende de algunas 
condiciones propias de la zona y sus costumbres, tal es el caso de condiciones climáticas, de almacenamiento, transporte y conservación. La humedad puede variar debido a dichas condiciones, alterando el PCI necesario para el aprovechamiento energético de los residuos.

Como ya se indicó con anterioridad, el contenido máximo de humedad de un residuo sólido debe ser de entre el 55 y el $60 \%$ para que sea considerado como materia prima para incineración. Los tipos de residuos: papel, cartón y madera presentaron contenidos de humedad necesarias para ser consideradas como materia prima para dichos procesos, mientras que materia orgánica superó el máximo establecido con un valor de $71 \%$, por lo tanto, este tipo de residuo no podría ser utilizado como materia prima para procesos de incineración, así como tampoco podría serlo la mezcla total de los RSU (muestra mixta), pues llega al 67,4\% de humedad.

La abundante humedad presente en la muestra mixta redujo el potencial energético de la misma; pese a ello, este parámetro no impide el aprovechamiento de los residuos. En cuanto a la muestra de textil, el porcentaje de humedad fue insignificante para la mayoría de la muestra, por lo cual, no influyó determinantemente en la obtención del poder calórico inferior, es decir, este parámetro no representa un limitante para el aprovechamiento energético de los RSU.

La caracterización de los RSU de la parroquia Limoncocha reveló una composición mayoritaria de materia orgánica (73\%); a pesar de ello, esto no representa un limitante para el aprovechamiento energético, a diferencia de la cantidad de residuos generados, la cual es insuficiente para establecer una planta de incineración, pues se produce diariamente apenas 1,7 toneladas de RSU.

El potencial energético que brindan los RSU de la parroquia Limoncocha, para todas las muestras analizadas, son aptos para someterlos a procesos de aprovechamiento energético con recuperación de energía, ya que en promedio el PCI supera las $3100 \mathrm{kcal} / \mathrm{kg}$ requeridas, por lo que, efectivamente, una incineración es una alternativa viable para la minimización de residuos, aunque debe ser planteada en un contexto más amplio al presentado en este estudio, que incluye los volúmenes de generación, la humedad y el contenido de metales en las cenizas de los residuos generados en el aprovechamiento energético. Todos estos datos disponibles en la UISEK, como parte de la línea de investigación de RSU. 
Considerando tanto las características fisicoquímicas de los residuos como su flujo anual, se descarta la incineración como sistema de gestión en la parroquia Limoncocha, ya que a pesar de que el PCI se encuentra dentro del rango recomendado y la composición y el porcentaje de humedad no fueron factores limitantes para la implementación de un sistema de incineración, la generación per cápita de apenas $0,61 \mathrm{kh} / \mathrm{hab}$. / día (Mora 2016) de los residuos en la parroquia resulta insuficiente, por lo que no se justifican los costos de operación y tratamiento de gases, agua y cenizas, lo que implica establecer una planta incineradora.

El presente estudio establece valores de PCS aptos para la valorización energética; sin embargo, esto tiene adicionalmente una connotación social, pues de acuerdo con la propuesta realizada por Almeida, Oviedo, Coral y Sevilla (2016), uno de los principales beneficios que se generaría de la gestión de residuos sólidos urbanos en Limoncocha es la posibilidad de crear microempresas que se dediquen a su tratamiento en reciclaje o recuperación para su comercialización o en el aprovechamiento energético de estos residuos.

Una opción para el aprovechamiento energético de residuos de pequeñas poblaciones es conformar mancomunidades, mismas que capten los RSU de varias pequeñas y medianas poblaciones, con el fin de generar un volumen de residuos suficiente para que el aprovechamiento energético sea representativo. De varios autores y especificaciones técnicas se recomienda un volumen de residuos mínimo de 17 ton / día. La decisión final estará a cargo del gobierno parroquial, así como de la comunidad en pleno, pues su compromiso es vital para este tipo de proyectos de manejo de RSU.

\section{Referencias}

Almeida Fernanda, Jorge Oviedo, Katty Coral y Silvia Sevilla. 2016. "Diseño del sistema de gestión de residuos sólidos urbanos que incluya una resolución de junta parroquial para la parroquia Limoncocha 2016". Tesis de maestría, UISEK, Quito. https://bit.ly/3krmHut. Alonso, Carlos, Elena Martínez y Jesús Olías. 2003. Manual para la gestión de los residuos urbanos. Madrid: Editora La Ley.

ASOKIL. 2015. Censo poblaciones de socios de la asociación ASOKIL. Limoncocha: Asociación ASOKIL.

Ayala, Inés, 2013. “Cuantificación del poder calórico superior e inferior de los residuos sóli- 
dos urbanos del Distrito Metropolitano de Quito". Tesis de grado, UISEK, Quito. https:// bit.ly/2FRVAK3.

Banco Internacional para el Desarrollo / Banco Mundial. 1999. Municipal Solid Waste Incineration. Washington D. C.: Banco Mundial.

Banco Mundial. 2018. "Informe del Banco Mundial: Los desechos a nivel mundial crecerán un $70 \%$ para 2050, a menos que se adopten medidas urgentes". Accedido 27 de agosto de 2020. https://bit.ly/2REM0Nv.

Barras, Francisco. 2008. "Residuos urbanos o municipales". Artículo EOI Escuela de Negocios: $17-18$. https://bit.ly/3ifxyXX.

Bastidas, Daniel. 2009. "Ficha informativa de los humedales de Ramsar (FIR)". Accedido 15 de abril. https://bit.ly/32DxMTf.

Cabrera, Juan. 2016. “Cuantificación del poder calórico superior e inferior de los residuos sólidos urbanos: papel, cartón, madera y materia orgánica de la parroquia de Limoncocha. 2015-2016". Tesis de grado, UISEK, Quito. https://bit.ly/33Bv7J2.

Cano, Leslie, Jorge Oviedo y Katty Coral. 2016. "Cuantificación del porcentaje de humedad y cenizas contenidos en los residuos urbanos de la parroquia de Limoncocha”. Tesis de grado, UISEK, Quito. https://bit.ly/3mvu0TZ.

Castillo, María José. 2012. "Determinación de la composición y densidad de los residuos sólidos urbanos del Distrito Metropolitano de Quito con fines de aprovechamiento energético y reducción de emisiones de gases de efecto invernadero". Tesis de grado, UISEK, Quito. https://bit.ly/3cc8GOk.

Climate-data Org. 2018. Clima Limoncocha. Accedido febrero de 2020. https://bit.ly/35NKKQb.

EC Instituto Nacional de Estadística y Censos (INEC). 2010. Censo de población y vivienda. Ecuador. Accedido junio de 2018. https://bit.ly/33CSVfM.

---. 2014. Ecuador en cifras: estadística de información ambiental económica en gobiernos autónomos descentralizados municipales. Accedido 16 de febrero de 2018. https://bit.ly/ 3bHvBAT.

EC Ministerio del Ambiente (EC MAE). 2015. Hitos en la gestión integral de residuos sólidos en Ecuador. Accedido 20 de septiembre. https://bit.ly/2FHtf9n.

European Union Energy Initiative (EUEI). 2008. Amazonía: energías renovables, electrificación rural y desarrollo humano sostenible. Accedido 26 de marzo. https://bit.ly/3mynDzi.

Garcés, Mauricio. 2016. "Cuantificación del porcentaje de humedad y cenizas contenidos en los residuos sólidos urbanos del Distrito Metropolitano de Quito, año 2015-2016". Tesis de grado, UISEK, Quito. https://bit.ly/3kntg16.

Gobierno Autónomo Descentralizado de la parroquia Limoncocha. 2011. Plan de desarrollo y ordenamiento territorial de parroquia Limoncocha. Limoncocha: Geográficas SIS.

Lomas José, Cipriano Urbano, José Merino y Luis Camarero. 2001. Valorización de la biomasa en el País Vasco. Bilbao: Universidad del País Vasco. 
López, Félix, Teresa Álvarez Centeno y Francisco José Alguacil. 2012. Aprovechamiento energético de residuos: el caso de los neumáticos fuera de uso. Accedido junio de 2020. https://bit.ly/2ZoE47d.

Marañón, Michelle. 2015. "Modelo de gestión integral de los residuos sólidos urbanos en la cabecera parroquial de Limoncocha". Tesis de grado, UISEK, Quito. https://bit.ly/33C69Jv.

Mazzilli, Andrea. 2014. "Validación del método de cuantificación del porcentaje de humedad y porcentaje de cenizas contenido en los residuos sólidos urbanos del Distrito Metropolitano de Quito 2013". Tesis de grado, UISEK, Quito. https://bit.ly/3ktQtyY.

Montenegro, María Belén. 2015. "Valoración y gestión económico ambiental para la Conservación de la Reserva Biológica Limoncocha, Shushufindi, Sucumbíos-Ecuador". Tesis de grado, UISEK, Quito. https://bit.ly/2Hbjwce.

Montiel-Bohórquez, Daniel Néstor, y Juan Pérez. 2019. “Generación de energía a partir de residuos sólidos urbanos: estrategias termodinámicas para optimizar el desempeño de centrales térmicas". Información Tecnológica 30 (1): 273-284. https://bit.ly/2RDbD1i.

Mora, Cecilia. 2016. "Diagnóstico del manejo integral de residuos sólidos urbanos de la cabecera parroquial de Limoncocha, mediante muestreos realizados en la población (20152016)". Tesis de grado. UISEK, Quito. https://bit.ly/2FMMG0E.

Morales Vallejo, Pedro. 2012. "Estadística aplicada a las ciencias sociales: tamaño necesario de la muestra: ¿Cuántos sujetos necesitamos?”. Accedido 22 de abril. https://bit.ly/32dCUx3.

Moratorio Diego, Ignacio Rocco y Marcelo Castelli. 2012. “Conversión de residuos sólidos urbanos en energía". Montevideo: Universidad de Montevideo.

Muñoz, Denis. 2019. "Propuesta de una planta incineradora de residuos sólidos urbanos en Santa Clara". Tesis de grado, Universidad Central de Las Villas, Santa Clara de Cuba. https://bit.ly/3cbwvGf.

Ninabanda, Andrea. 2016. "Cuantificación del poder calórico superior e inferior de los residuos sólidos urbanos: textil y mixto de la parroquia Limoncocha. 2015-2016". Tesis de grado, UISEK, Quito. https://bit.ly/2FJPkEq.

Pacheco, Frank. 2016. "Cuantificación del poder calórico superior e inferior de los residuos sólidos urbanos de Limoncocha. Año 2015". Tesis de grado, UISEK, Quito. https://bit. ly/3heGD1T.

Ribadeneira, Joe. 2014. "Cuantificación del poder calórico superior e inferior de los residuos sólidos urbanos del Distrito Metropolitano de Quito. Año 2013-2014". Tesis de grado, UISEK, Quito. https://bit.ly/2FNv0SB.

Romero, Arturo. 2010. "La incineradora de residuos: ¿está justificado el rechazo social?". Revista Real Academia de Ciencias Exactas, Físicas y Naturales 104 (1): 175-187. https:// bit.ly/3hEM7mQ.

Sakurai, Kunitoshi. 2000. Guía HDT 17: método sencillo del análisis de residuos sólidos. Lima: Centro Panamericano de Ingeniería Sanitaria y Ciencias del Ambiente (CEPIS). https://bit.ly/3iG0bO4. 